\title{
Releasing control
}

DOI:

10.1038/nrn2108

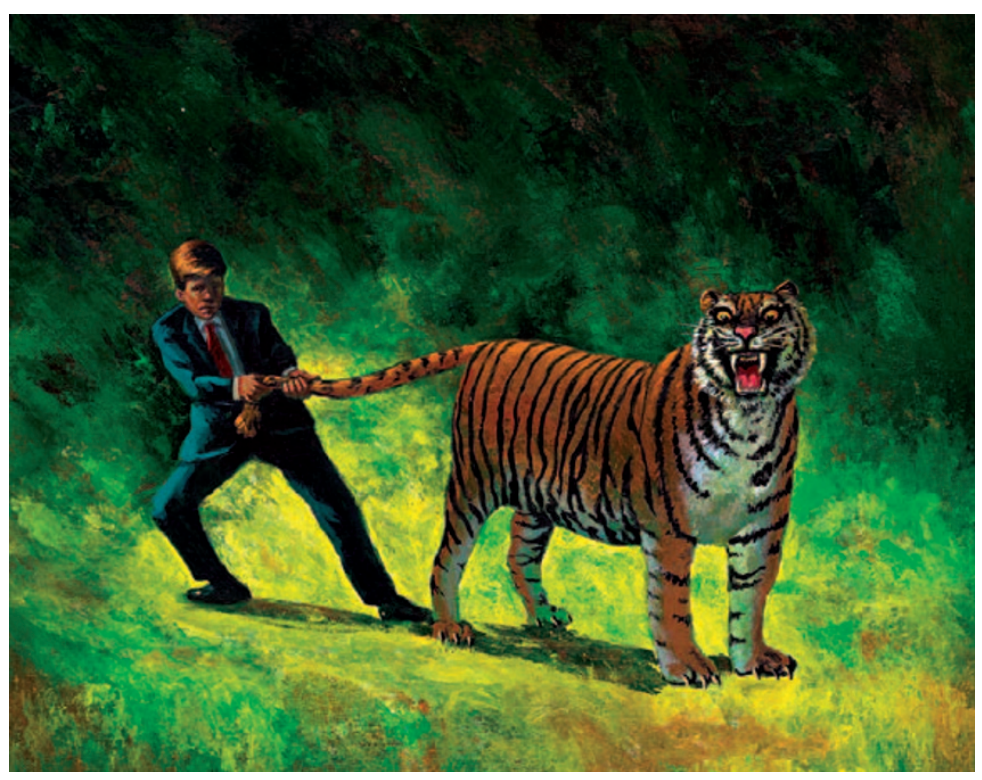

Symmetric or asymmetric neuroepithelial cell division is accompanied by the equal and unequal inheritance, respectively, of the apical plasma membrane domain by daughter cells. Around the onset of neurogenesis and the switch from mainly symmetric to mainly asymmetric divisions, neuroepithelial cells reduce the size of the apical plasma membrane domain. Dubreuil et al. show that this is achieved in part by releasing some of the apical membrane into the extracellular space.

Prominin 1 (PROM1), a component of the neuroepithelial apical plasma membrane, has been found in small particles that accumulate in the neural tube lumen at the onset of neurogenesis. The authors used time-lapse imaging of the embryonic chick spinal cord to investigate the formation of these particles, revealing that they are released from the apical surface of neuroepithelial cells.

The authors set out to determine the precise site of origin of the particles. They found a high concentration of PROM1 at the midbody, a bridge that forms during mitosis at the apical surface and connects two daughter cells just before their final separation. Furthermore, they found that PROM1-containing extracellular particles contained anillin, a characteristic component of the midbody, indicating that the midbody might be the source of the particles. Indeed, time-lapse imaging of dividing neuroepithelial cells expressing a green fluorescent protein (GFP)anillin fusion protein revealed its release in the form of small particles from the apical surface. In addition, the authors showed that PROM1 is also localized to primary cilia of neuroepithelial cells around the time of neurogenesis, providing another possible source of membrane release.

To investigate the relationship between particle release and the type of cell division, the authors used mice that express GFP in cells undergoing neurogenic, asymmetric divisions. They showed that apically localized midbodies are mainly found in GFPnegative, symmetrically dividing cells. This indicates that the release of PROM1 and anillin-containing particles from the midbody took place only in cells that were undergoing symmetric divisions.

This study shows that, around the onset of neurogenesis, symmetrically dividing neuroepithelial cells release apical membrane from the midbody and primary cilia. The significance of this event remains to be elucidated; however, as PROM1 is a somatic stem cell marker, by discarding this microdomain the cell might influence future cell fate decisions. Alternatively, this might provide a method of signalling to neighbouring neuroepithelial cells.

Katherine Whalley

ORIGINAL RESEARCH PAPER Dubreuil, V., Marzesco, A.-M., Corbeil, D., Huttner, W. B. \& Wilsch-Brauninger, M. Midbody and primary cilium of neural progenitors release extracellular membrane particles enriched in the stem cell marker prominin-1.J. Cell Biol. 5 Feb 2007 (doi:10.1083/jcb.200608137) 